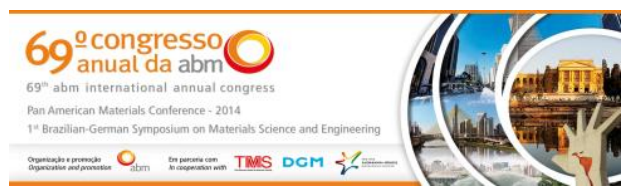

Tema: Mecânica da Fratura e Integridade Estrutural

\title{
AVALIAÇÃO EXPERIMENTAL DAS PROPRIEDADES TENSÃO- DEFORMAÇÃO DE UM AÇO API 5L X65 SOUR APÓS DIFERENTES TEMPOS DE IMERSÃO EM SOLUÇÃO SATURADA POR $\mathrm{H}_{2} \mathrm{~S}^{*}$
}

\section{Resumo}

Brunno Spinelli Pimentel ${ }^{1}$ Duberney Hincapie-Ladino² Neusa Alonso-Falleiros ${ }^{3}$ Rodrigo Magnabosco 4

Ambientes contendo $\mathrm{H}_{2} \mathrm{~S}$ e $\mathrm{CO}_{2}$ impõem condições severas a componentes fabricados em aços, já que estes podem sofrer corrosão com perda de propriedades mecânicas e falha das instalações. Uma preocupação é a quantificação dos fenômenos envolvendo difusão do hidrogênio atômico, o qual (seja pelo gás $\mathrm{H}_{2}$ em interfaces ou pela interação com ligações atômicas ou discordâncias) pode causar redução nas energias superficiais de fraturamento prejudicando ductilidade e tenacidade. Neste cenário, este trabalho investiga a fragilização por hidrogênio em um aço microligado API $5 \mathrm{~L}$ X65 sour obtido de um tubo de diâmetro externo 32" e espessura de parede $20,6 \mathrm{~mm}$ fabricado pelo processo UOE. Amostras longitudinais e transversais de tração (ASTM E8/E8M) foram extraídas e imersas em solução saturada com $\mathrm{H}_{2} \mathrm{~S}$. $\mathrm{O}$ eletrólito empregado foi a solução $A$ (ácido acético contendo cloreto de sódio) da norma NACE TM02842011, fazendo-se purga com $\mathrm{N}_{2}$ e injeções de $\mathrm{H}_{2} \mathrm{~S}$. Os tempos de imersão foram de 0, 96 e 360 horas, sendo os ensaios de tração realizados após doze dias do término da imersão. Os resultados não revelaram efeito estatisticamente relevante na resistência mecânica, na evolução tensão-deformação e tampouco no módulo de tenacidade. Entretanto, foi observado maior espalhamento dos resultados advindos de amostras submetidas a $\mathrm{H}_{2} \mathrm{~S}$. Esse comportamento sugere que o hidrogênio influenciou no micromecanismo local de fraturamento, mas com inexpressivo comprometimento das propriedades monotônicas macroscópicas. Tal desempenho pode ser fruto da microestrutura com grãos refinados e do baixo nível de inclusões encontrado, indicando poucos e uniformes sítios de ancoramento de hidrogênio irreversível.

Palavras-chave: Aços microligados; API-5L-X65; Ambiente sour; Corrosão; Propriedades à tração.

\section{EXPERIMENTAL ASSESSMENT OF TENSILE PROPERTIES OF AN API 5L X65 SOUR STEEL AFTER DIFFERENT TIMES OF IMMERSION IN A $\mathrm{H}_{2}$ S SATURATED SOLUTION}

\begin{abstract}
Sour service environments containing $\mathrm{H}_{2} \mathrm{~S}$ and $\mathrm{CO}_{2}$ impose severe operating conditions to steel-made components, since they can be susceptible to corrosion damage leading to losses on mechanical properties and consequently failures. One key concern is related to the diffusion of atomic hydrogen, which (due to $\mathrm{H}_{2}$ gas in interfaces or due to the interaction with atomic bonds or dislocations) can reduce energies required for fracture and therefore reduce ductility and toughness. In this context, this work examines the hydrogen embrittlement in a microalloyed API-5L-X65 sour steel obtained from a UOE pipe with OD $=32$ " and $t=20.6$ $\mathrm{mm}$. Tensile specimens according to ASTM E8/E8M where extracted from the longitudinal and transverse directions and were exposed to a $\mathrm{H}_{2} \mathrm{~S}$ saturated solution. The selected electrolyte was solution $\mathrm{A}$ (acetic acid containing sodium chloride) from NACE TM0284-2011 standard, purged with $\mathrm{N}_{2}$, with injection of $\mathrm{H}_{2} \mathrm{~S}$. Immersion times comprehended 0, 96 and 360 hours and the tensile tests were performed 12 days after the end of the exposure. The obtained results revealed a negligible effect on mechanical strength, stress-strain evolution and toughness. However, in all cases a larger scatter on mechanical properties was found for corroded samples. This behavior indicates that hydrogen affected the local micromechanism for fracture, but with negligible effects on the macroscopic monotonic mechanical properties. This performance can be a result of the refined microstructure combined to the low incidence of inclusions found in the studied steel, which can represent a small quantity of uniformly distributed sites for irreversible hydrogen locking.
\end{abstract}

Keywords: Microalloyed steels; API-5L-X65; Sour Service; Corrosion; Tensile properties.

1 Engenheiro de Materiais, Mestrando do Programa de Engenharia Mecânica, Centro Universitário da FEl, São Bernardo do Campo, SP, Brasil; brunnospinelli@gmail.com.

2 Engenheiro Mecânico, Doutorando, Programa de Engenharia Metalúrgica e de Materiais, EPUSP, São Paulo, SP, Brasil.

3 Engenheira Metalurgista, Doutora, Docente do Departamento de Engenharia Metalúrgica e de Materiais, EPUSP, São Paulo, SP, Brasil; nealonso@usp.br.

4 Engenheiro Metalurgista, Doutor, Docente, Departamento de Engenharia de Materiais do Centro Universitário da FEl, São Bernardo do Campo, SP, Brasil; rodrmagn@fei.edu.br.

5 Engenheiro Mecânico, Doutor, Docente, Departamento de Engenharia Mecânica, Centro Universitário da FEl, São Bernardo do Campo, SP, Brasil, gdonato@fei.edu.br.

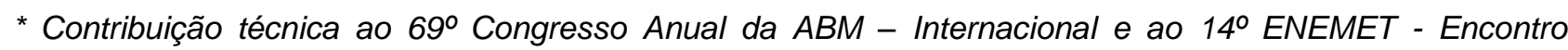
Nacional de Estudantes de Engenharia Metalúrgica, de Materiais e de Minas,21 a 25 de julho de 2014, São Paulo, SP, Brasil. 


\section{INTRODUÇÃO}

Na última década o Brasil tem sido palco de um expressivo incremento no consumo de energia. Como decorrência de sua matriz energética fortemente dependende do petróleo e gás, automaticamente existe grande pressão para o aumento na exploração e beneficiamento de gás natural, petróleo e seus derivados. Oportunamente, o cenário quantitativo dos recursos é favorável, já que as reservas provadas são $50 \%$ maiores nos dias atuais do que há dez anos, totalizando no ano de 2012 15,3 bilhões de barris de óleo e 450 bilhões de $\mathrm{m}^{3}$ de gás natural [1]. Entretanto, 94\% das reservas de óleo e $84 \%$ das reservas de gás estão localizadas no mar e boa parte destas na chamada camada présal; considerando por exemplo o campo de Tupi na bacia de Santos, a lâmina d'água ultrapassa os 2500 metros e a profundidade total dos poços atinge 7000 metros [2]. Como resultado, os componentes de exploração devem operar em águas ultraprofundas, com elevadas pressões e temperaturas e ambientes contendo acido sulfídrico $\left(\mathrm{H}_{2} \mathrm{~S}\right)$ e $\mathrm{CO}_{2}$, contando com mínimas possibilidades de manutenção ao longo da vida útil. Tais condições adversas podem acarretar corrosão com perda de propriedades mecânicas e decorrente falha das instalações.

A maior parte das atividades de exploração e transporte de gás natural e petróleo é realizada por meio de sistemas dutoviários compostos por dutos fabricados em aços microligados que atendem aos requisitos da norma API 5L [3], sendo os mais usuais fabricados com as classes $\mathrm{X} 65, \mathrm{X} 70$ e $\mathrm{X} 80$ [4]. Os ambientes agressivos citados anteriormente fazem com que tais classes de materiais tenham que ser adequadas para operação em ambientes ácidos (sour service), evitando ou minimizando efeitos como fratura induzida por hidrogênio (do inglês Hydrogen Induced Cracking - HIC) e corrosão sob tensão na presença de sulfeto (do inglês Sulfide Stress Cracking - SSC) [5]. Adequado desempenho sob tais condições só é conseguido por meio de materiais com microestrutura refinada, baixa incidência de inclusões e segregação, baixos teores de enxofre e fósforo e processamento termomecânico controlado (em termos de laminação e resfriamento controlados), como pode ser observado em detalhes nos trabalhos de Jung e colaboradores [6] e Siciliano e colaboradores [7].

Uma das preocupações centrais é a caracterização dos fenômenos envolvendo difusão do hidrogênio atômico, o qual (seja por moléculas de hidrogenio em interfaces ou pela interação com ligações atômicas ou discordâncias) pode causar redução nas energias superficiais de fraturamento prejudicando ductilidade e tenacidade. A principal fonte de átomos de hidrogênio é a reação catódica do mecanismo de corrosão de ferro em solução ácida; na presença de $\mathrm{H}_{2} \mathrm{~S}$ a formação de $\mathrm{H}_{2}$ é retardada, assim, grande parte dos átomos de $\mathrm{H}$ adsorvidos na superfície se difunde no metal [8], sendo que o hidrogênio atômico (o qual possui grande difusividade no aço), pode permanecer em solução sólida ou recombinar-se em hidrogênio molecular $\left(\mathrm{H}_{2}\right)$. Esta combinação usualmente se dá em torno de inclusões (p.ex.: MnS), bandas de segregação e descontinuidades da matriz do metal. A pressão resultante da formação de gás é elevada e pode resultar em plastificação e microtrincamento localizados [7]. Muitos ensaios para caracterização do efeito da fragilização por hidrogênio são conduzidos no próprio meio agressivo com hidrogênio atuante durante a deformação, como no trabalho de Nanninga e colaboradores [9], enquanto em outros casos os ensaios são realizados em amostras pré-carregadas com hidrogênio, a fim de avaliar o efeito do chamado hidrogênio irreversível. O segundo caso é o interesse desse trabalho.

Neste cenário, este trabalho investiga a fragilização por hidrogênio em um aço microligado API 5L X65 sour obtido de um tubo de 32" e espessura de parede 20,6 mm fabricado pelo processo UOE. Amostras longitudinais e transversais de tração foram

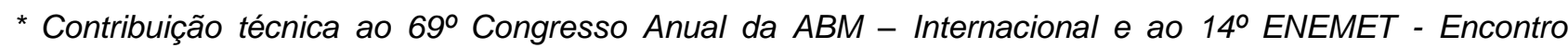
Nacional de Estudantes de Engenharia Metalúrgica, de Materiais e de Minas,21 a 25 de julho de 2014, São Paulo, SP, Brasil.
} 


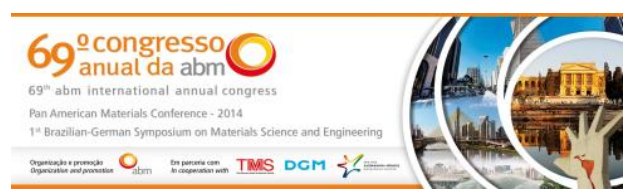

elaboradas segundo a norma ASTM E8/E8M [10] e expostas a $\mathrm{H}_{2} \mathrm{~S}$ utilizando a solução $A$ da norma NACE TM0284-2011 [11] por tempos de 0, 96 e 360 horas.Os ensaios de tração foram realizados após doze dias do término da imersão. Os resultados foram pósprocessados para a obtenção das propriedades mecânicas monotônicas e as fraturas foram submetidas a análises fractográficas por microscopia eletrônica de varredura para evidenciar o efeito do ataque químico no micromecanismo de falha.

\section{MATERIAIS E MÉTODOS}

No presente trabalho, foram utilizadas amostras de um aço de Alta Resistência e Baixa Liga (ARBL) classe API 5L X65 sour [3] com composição química apresentada pela Tabela 1. Vale ressaltar que o aço estudado foi fabricado com controle do fósforo e do enxofre e passou por tratamento das inclusões com cálcio; desta maneira, objetiva aplicações em ambientes ácidos contendo $\mathrm{H}_{2} \mathrm{~S}$ e as conclusões do trabalho devem ser avaliadas à luz de materiais com estas características. O material estudado apresenta microestrutura bastante refinada composta de ferrita e perlita (Figura 1a), com pequena quantidade de inclusões distribuídas de maneira homogênea na matriz, sendo categorizado como D1 de acordo com a norma ASTM E45 [12]. Ainda, foi evidenciada pelos autores a presença de cementita nos contornos de grão (especialmente nos pontos triplos) e não foi encontrada segregação central próxima da meia espessura da chapa.

Tabela 1. Composição Química do aço API-5L-X65 sour [3] utilizado no estudo.

\begin{tabular}{ccccccccccccc}
\hline & $\mathrm{C}$ & $\mathrm{S}$ & $\mathrm{P}$ & $\mathrm{Al}$ & $\mathrm{Si}$ & $\mathrm{Cu}$ & $\mathrm{Cr}$ & $\mathrm{Mn}$ & $\mathrm{Ni}$ & $\mathrm{Mo}$ & $\mathrm{N}$ & $\mathrm{V}+\mathrm{Ti}+\mathrm{Nb}$ \\
\hline X65-sour & 0,040 & 0,001 & 0,010 & 0,040 & 0,350 & 0,050 & 0,030 & 1,370 & 0,060 & 0,020 & 0,004 & 0,070 \\
\hline
\end{tabular}

Todas as amostras para ensaios mecânicos foram extraídas do metal de base de um tubo fabricado por conformação a frio empregando o processo UOE [13], cujas dimensões principais podem ser encontradas na Tabela 2.

Tabela 2. Dimensões do tubo usado na extração das amostras.

\begin{tabular}{cccc}
\hline Identificação & $\begin{array}{c}\text { Diâmetro } \\
\text { (pol. / mm) }\end{array}$ & $\begin{array}{c}\text { Espessura } \\
(\mathrm{mm})\end{array}$ & $\begin{array}{c}\text { Comprimento } \\
(\mathrm{m})\end{array}$ \\
\hline Tubo X65 & $32 " / 813$ & 20,6 & 12 \\
\hline
\end{tabular}

As amostras de tração foram extraídas da meia espessura da parede do tubo e seguindo as recomendações dimensionais da norma ASTM E8/E8M [10]. A Figura 1(b) apresenta as dimensões principais da amostra desenvolvida para o estudo e o acabamento da região sensível foi o equivalente a usinagem fina.

Uma vez usinadas as amostras, um lote foi reservado para ensaios de tração sem exposição ao ambiente ácido (tais amostras são chamadas de 0h no trabalho). As demais amostras foram submetidas a imersões em dois tempos distintos (96h e 360h), a fim de se verificar o efeito nas propriedades à tração. $O$ eletrólito selecionado foi a solução $\mathrm{A}$ da norma ANSI/NACE TM0284 [11], que consiste em 5\% NaCl (cloreto de sódio) e 0,5\% $\mathrm{CH}_{3} \mathrm{COOH}$ (ácido acético) em água destilada, desaerada e saturada por $\mathrm{H}_{2} \mathrm{~S}$. A solução foi preparada num recipiente separado, transferida até um recipiente onde foi realizada a purga da solução com $\mathrm{N}_{2}$ durante uma hora, com uma vazão de $100 \mathrm{~mL} / \mathrm{min}$ por litro de solução. Os corpos de prova foram posicionados na célula de ensaio sem que tivessem contato entre si. A célula de ensaio foi selada e preenchida com 5 litros de solução do recipiente de purga; nesse momento, foi feita uma nova purga com nitrogênio durante 1 hora com uma vazão de $100 \mathrm{~mL} / \mathrm{min}$ por litro de solução; após o preenchimento, foi borbulhado $\mathrm{H}_{2} \mathrm{~S}$ na solução com uma vazão de $200 \mathrm{~mL} / \mathrm{min}$ por litro de solução durante 1

\footnotetext{
* Contribuição técnica ao 69ํ Congresso Anual da ABM - Internacional e ao 14ํㅡㄹ ENEMET - Encontro Nacional de Estudantes de Engenharia Metalúrgica, de Materiais e de Minas,21 a 25 de julho de 2014, São Paulo, SP, Brasil.
} 


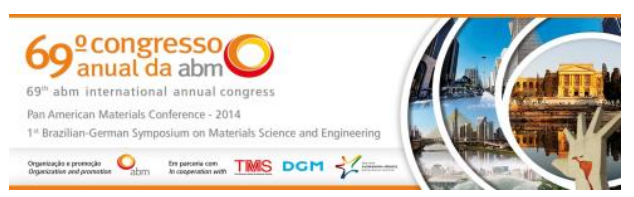

hora. A duração da primeira imersão foi de $96 \mathrm{~h}$ e da segunda de $360 \mathrm{~h}$, ambas contadas a partir do final da injeção de $\mathrm{H}_{2} \mathrm{~S}$. A temperatura da solução foi mantida em $25^{ \pm} 3^{\circ} \mathrm{C}$. Após a finalização da imersão, as amostras foram extraídas da célula de ensaio, lavadas com detergente e álcool e acondicionadas em dessecador. A Tabela 3 apresenta a matriz de experimentos conduzidos no trabalho. Para o material em condição original de fornecimento, 3 amostras foram suficientes para a adequada caracterização; para 96h e 360h, por sua vez, foram ensaiadas respectivamente 10 e 5 peças em cada direção, resultando total de 36 ensaios.

Tabela 3. Amostras ensaiadas sob tração para a realização do trabalho.

\begin{tabular}{ccc}
\hline $\begin{array}{c}\text { Tempo de exposição } \\
(\mathrm{h})\end{array}$ & $\begin{array}{c}\text { Amostras } \\
\text { longitudinais }\end{array}$ & $\begin{array}{c}\text { Amostras } \\
\text { transversais }\end{array}$ \\
\hline 0 & 3 & 3 \\
\hline 96 & 10 & 10 \\
\hline 360 & 5 & 5 \\
\hline
\end{tabular}

Entre a imersão e a realização dos ensaios de tração, houve um intervalo de 12 dias, ao longo dos quais as amostras permaneceram em um dessecador dedicado. Os ensaios de tração foram realizados seguindo as recomendações da norma ASTM E8/E8M [10] em uma máquina universal de ensaios MTS 810, de capacidade máxima $250 \mathrm{kN}$ e utilizando um extensômetro MTS 634.12-54 de abertura inicial 25 mm. A velocidade de solicitação foi de $0,05 \mathrm{~mm} / \mathrm{min}$ no regime elástico e $0,20 \mathrm{~mm} / \mathrm{min}$ após o escoamento. A Figura 1(c) apresenta uma imagem real de uma amostra não exposta a $\mathrm{H}_{2} \mathrm{~S}$ fixada para ensaio. Todas as amostras foram pintadas com uma fina camada de tinta de traçagem azul e foram ensaiadas com um fundo branco, possibilitando a aquisição de imagens de alta resolução em tempo real para a visualização em detalhes da estricção das amostras. Todo o pós-processamento dos dados de ensaio foi realizado em rotina especificamente desenvolvida usando linguagem MatLab, permitindo a determinação de propriedades de resistência, ductilidade, evolução tensão-deformação e o módulo de tenacidade.

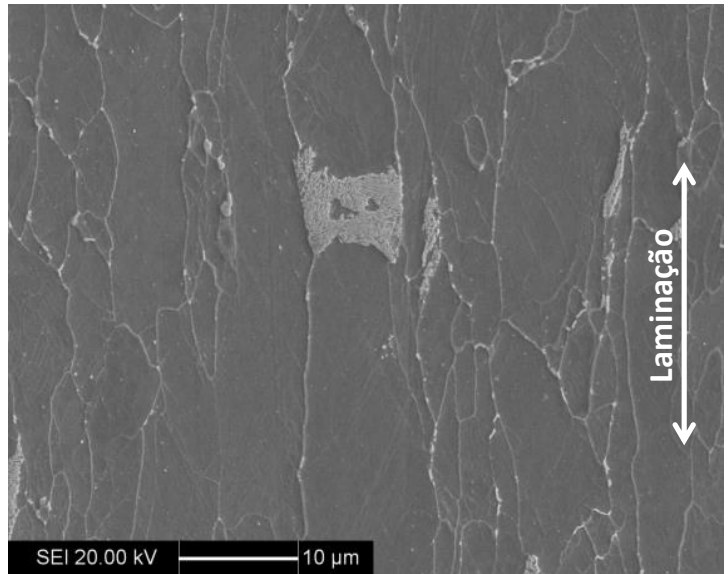

(a)

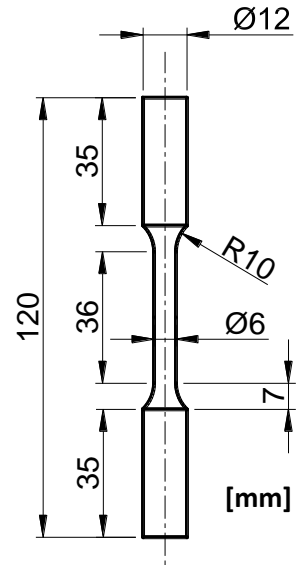

(b)

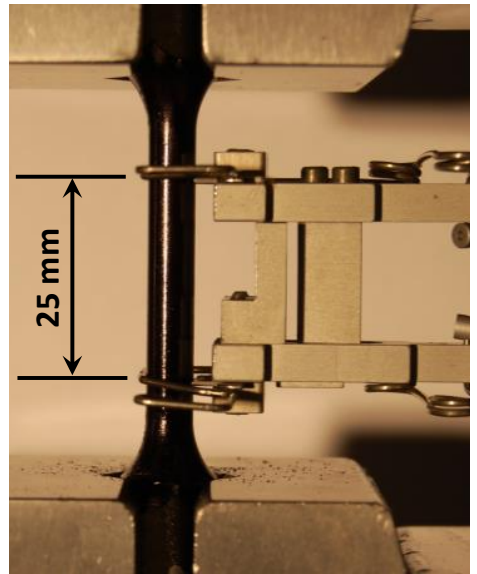

(c)

Figura 1. (a) Micrografia do aço X65 estudado, (b) Corpo de prova de tração segundo ASTM E8/E8M e (c) amostra pronta para ensaio em máquina MTS 810 de $250 \mathrm{kN}$ com extensômetro de $25 \mathrm{~mm}$.

Após a conclusão dos ensaios de tração, amostras selecionadas tiveram as fraturas e seções transversais examinadas por Microscopia Eletrônica de Varredura (MEV) nas facilidades do Centro de Desenvolvimento de Materiais Metálicos da FEI (CDMatM-FEI). O objetivo central foi a identificação do micromecanismo de fratura (dúctil ou frágil) e

\footnotetext{
* Contribuição técnica ao 69ํ Congresso Anual da ABM - Internacional e ao 14ํㅡㄹ ENEMET - Encontro Nacional de Estudantes de Engenharia Metalúrgica, de Materiais e de Minas,21 a 25 de julho de 2014, São Paulo, SP, Brasil.
} 


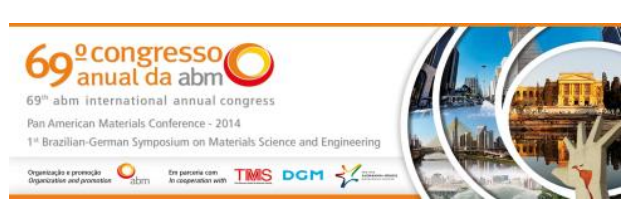

eventual identificação de microfraturamentos transversais (separações) nas proximidades da seção de fratura após a exposição do material ao ambiente agressivo contendo $\mathrm{H}_{2} \mathrm{~S}$.

\section{RESULTADOS E DISCUSSÃO}

Os resultados centrais do presente trabalho derivam da análise das curvas tensãodeformação (em suas vertentes de engenharia e verdadeiras), além da análise postmortem das amostras fraturadas para identificação do micromecanismo da falha e eventuais comportamentos anômalos advindos da absorção de hidrogênio a partir de uma solução aquosa contendo $\mathrm{H}_{2} \mathrm{~S}$. Tais resultados são apresentados na sequência, acompanhados da respectiva discussão.

\subsection{Curvas tensão-deformação}

A Figura 2 apresenta as evoluções de tensão de engenharia $(S)$ versus deformação de engenharia (e) para orientações longitudinal (Figura 2a) e transversal (Figura 2b) considerando diferentes tempos de imersão do material no ambiente ácido. Devido à pequena variação do comportamento $S$-e entre diferentes corpos de provas de uma mesma amostra, as curvas apresentadas representam uma curva selecionada para cada condição. Fica evidente um reduzido efeito do tempo de imersão nas propriedades macroscópicas do material estudado; somente a deformação de fratura apresentou ligeira alteração, mas sem tendência definida ou representatividade estatística. Para um melhor entendimento das propriedades mecânicas obtidas, a seção seguinte apresenta a quantificação de cada propriedademecânica avaliada.
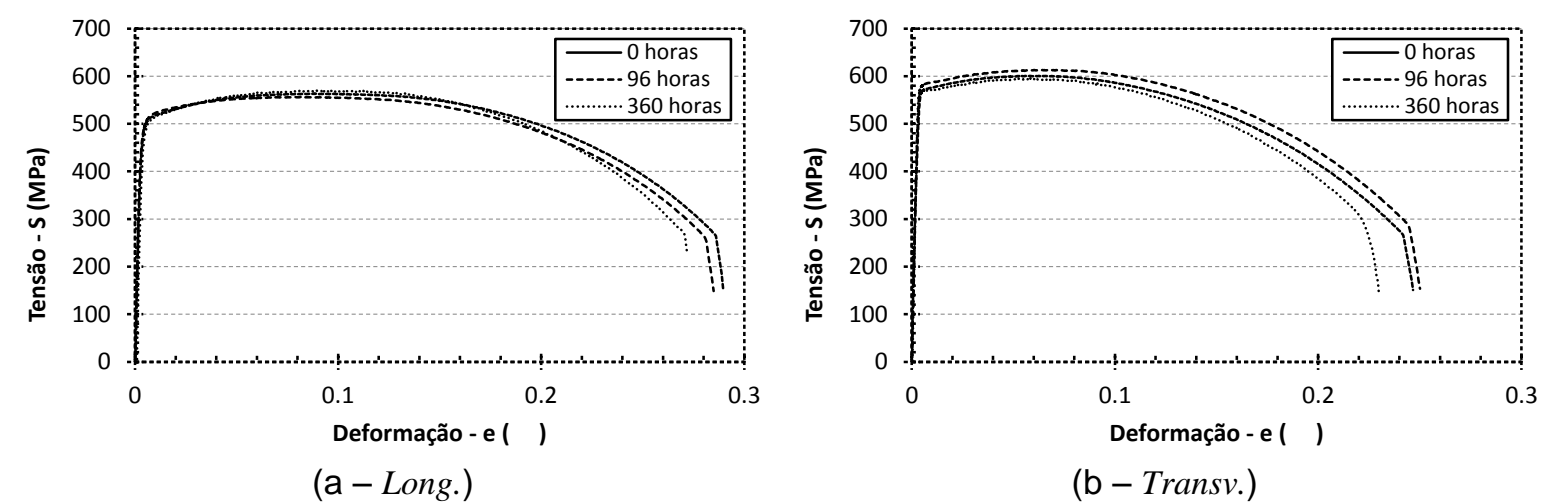

Figura 2.Curvas tensão-deformação representativas do aço API-5L-X65 sour para diferentes tempos de exposição a $\mathrm{H}_{2} \mathrm{~S}$ a partir de (a) amostras longitudinais e (b) amostras transversais.

\subsection{Propriedades mecânicas monotônicas}

A Figura 3(a) apresenta o efeito de diferentes tempos de imersão em ambiente ácido na tensão limite de escoamento $\left(S_{L E}\right)$ do aço API $5 \mathrm{~L}$ X65 sour estudado. Para a obtenção do $S_{L E}$, foi empregada a técnica de $0,2 \%$ offset recomendada pela ASTM E8/E8M [10]. Para maior precisão, a inclinação do regime elástico que serve de base para o método foi determinada por um algoritmo de otimização baseado em coeficiente de múltipla correlação. Os resultados demonstram anisotropia de propriedades ao escoamento, mas revelam que não houve efeito estatisticamente relevante dos ataques no limite de escoamento do material, tanto na direção longitudinal como na transversal. Entretanto, vale mencionar o expressivo aumento no espalhamento experimental dos resultados das amostras atacadas, mesmo com maior número de corpos de provas em relação à condição original (0h). Esta ocorrência pode indicar um efeito do hidrogênio irreversível

\footnotetext{
* Contribuição técnica ao 69ํㅡㄴ Congresso Anual da ABM - Internacional e ao 14 ENEMET - Encontro Nacional de Estudantes de Engenharia Metalúrgica, de Materiais e de Minas,21 a 25 de julho de 2014, São Paulo, SP, Brasil.
} 

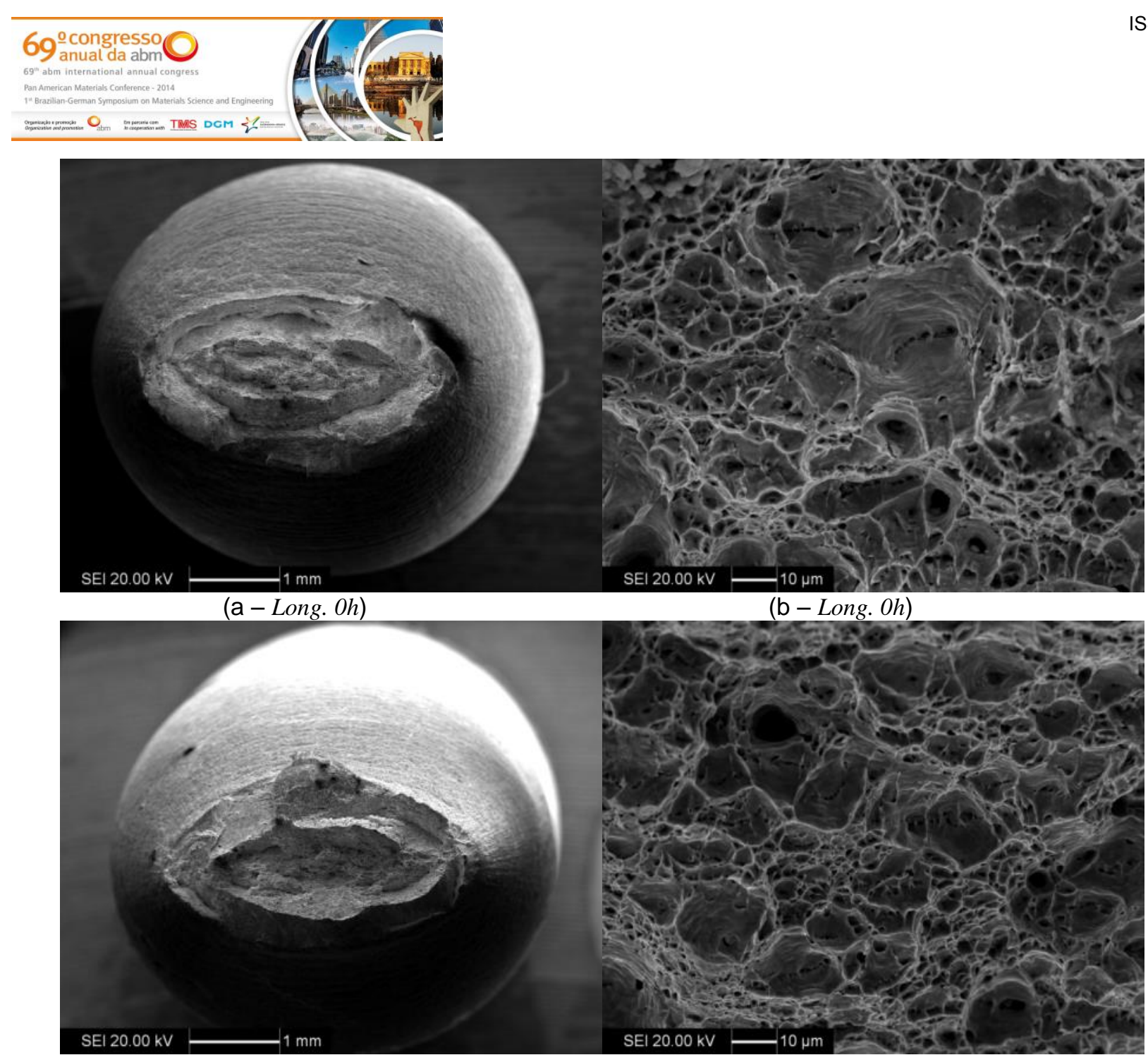

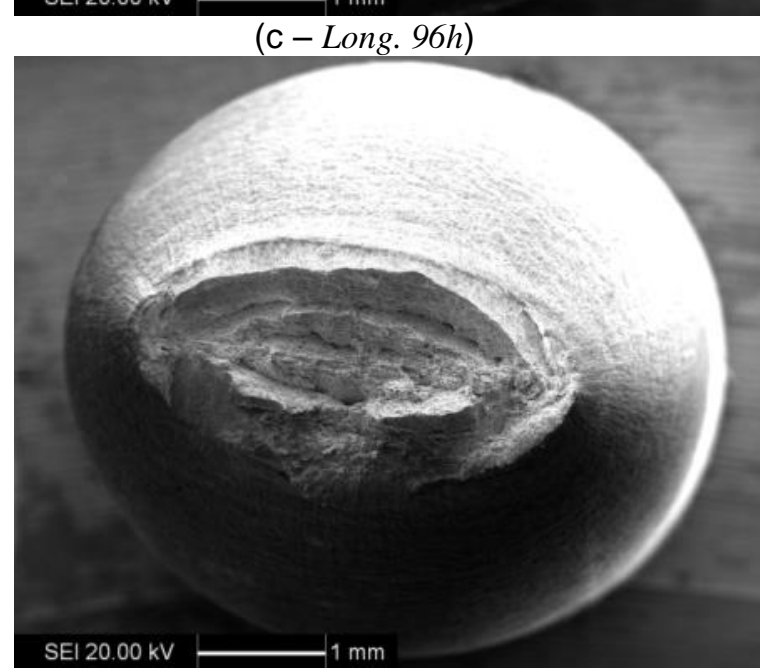

(e- Long. 360h) $(\mathrm{d}-$ Long. $96 h)$

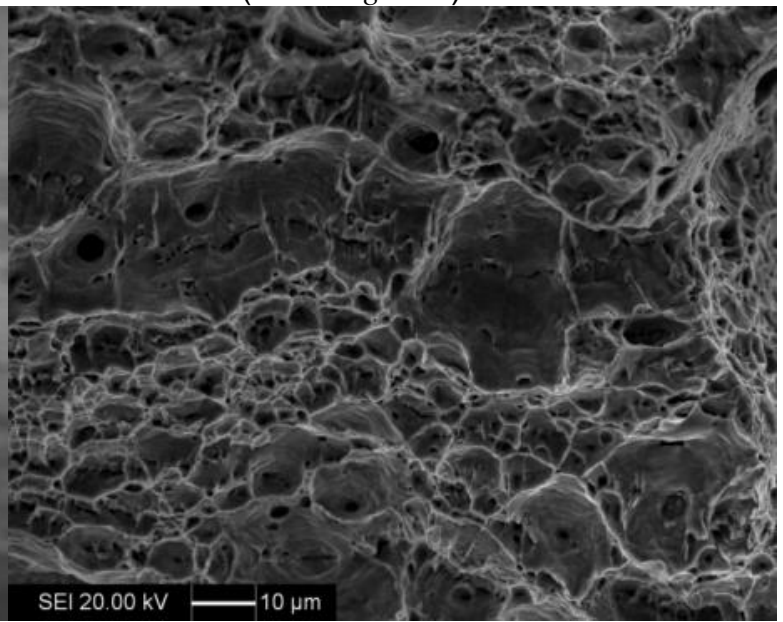

(f - Long. 360h)

Figura 6.Morfologias das fraturas obtidas para amostras longitudinais expostas a diferentes tempos de imersão em ambiente ácido.

\footnotetext{
* Contribuição técnica ao 69ํㅡㄹ Congresso Anual da ABM - Internacional e ao 14ํㅡㄹ ENEMET - Encontro Nacional de Estudantes de Engenharia Metalúrgica, de Materiais e de Minas,21 a 25 de julho de 2014, São Paulo, SP, Brasil.
} 

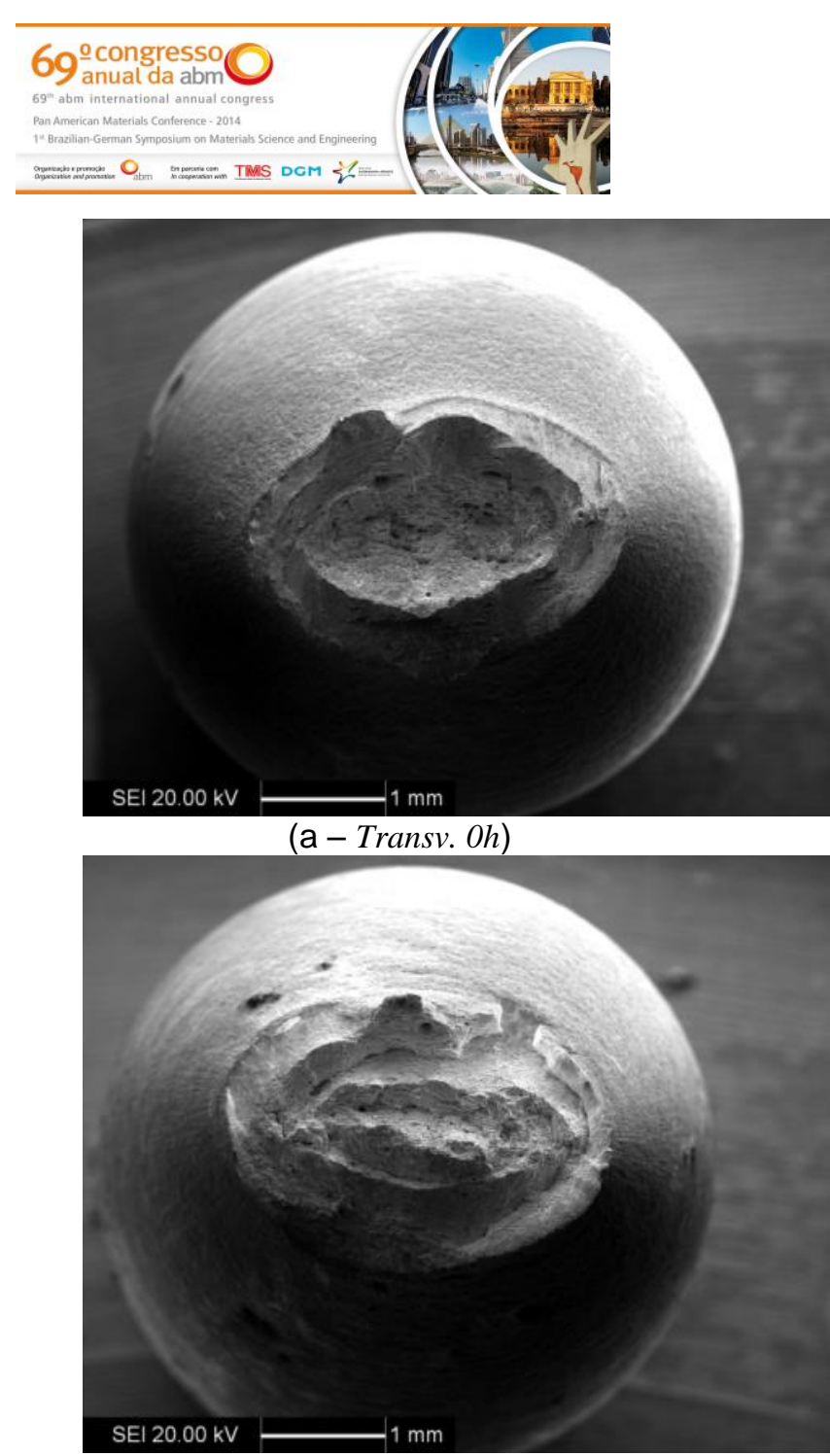

(c-Transv. 96h)

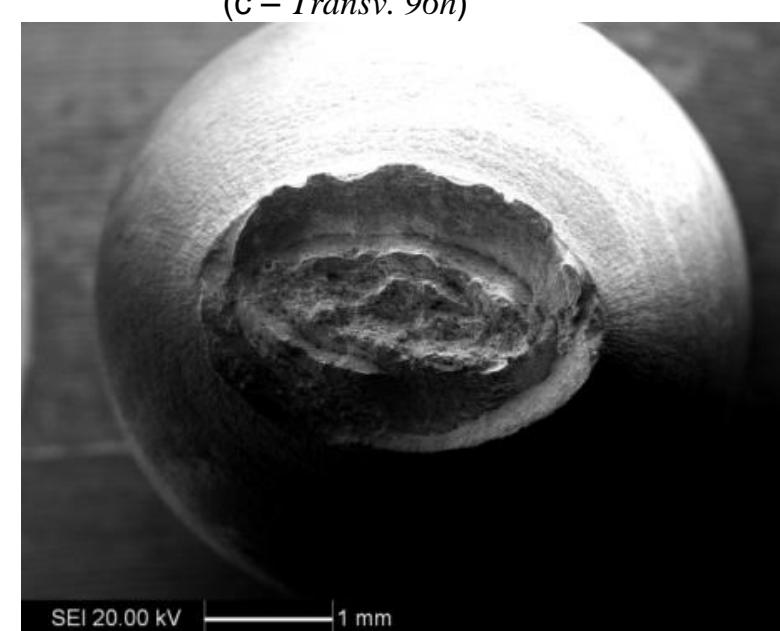

(e - Transv. 360h)

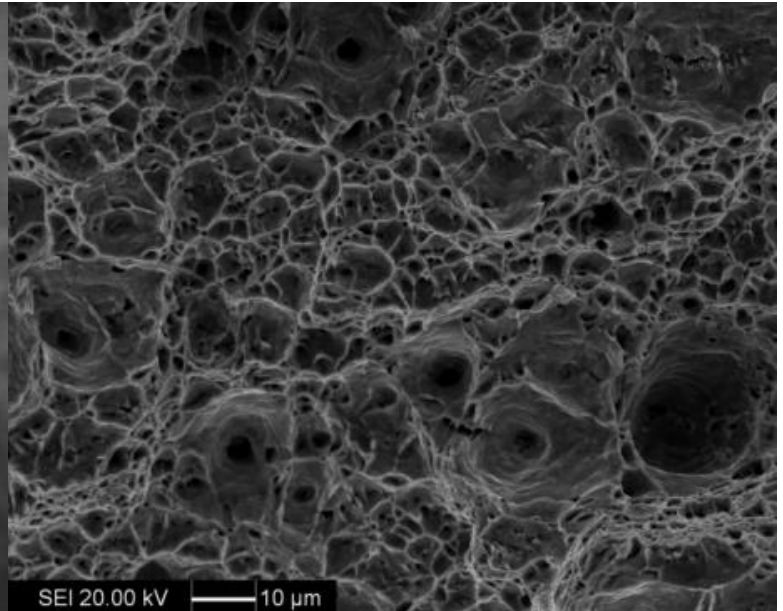

SEl $20.00 \mathrm{kV}=(\mathrm{b}-$ Transv. Oh)

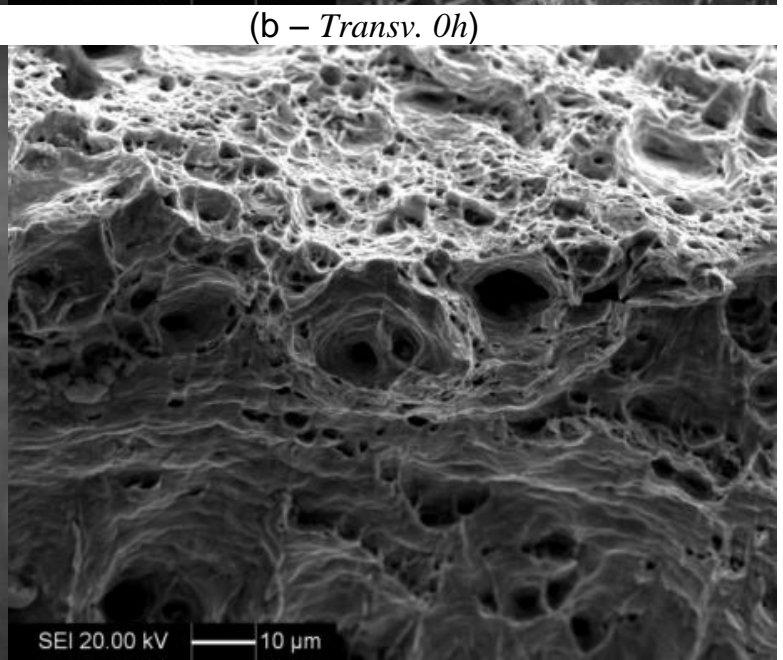

$(\mathrm{d}-$ Transv, $96 \mathrm{~h})$

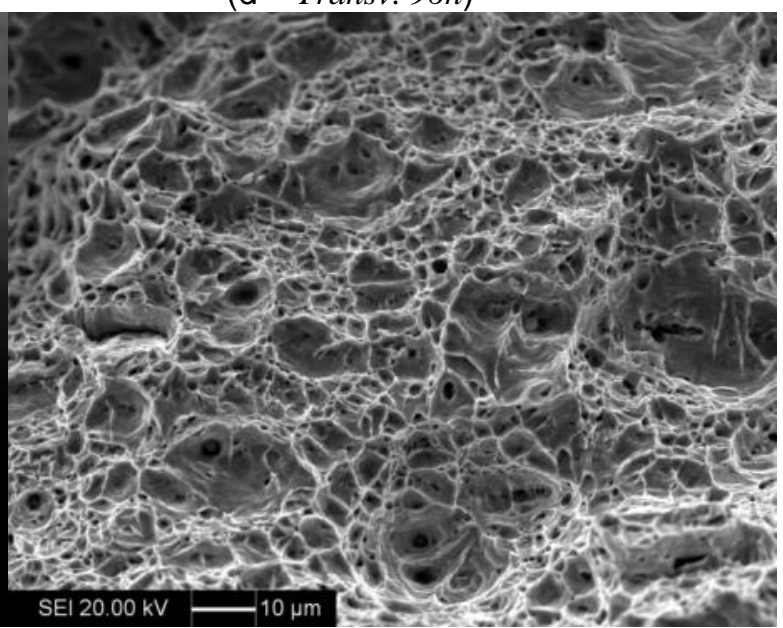

$(\mathfrak{f}-$ Transv. 360h)

Figura 7.Morfologias das fraturas obtidas para amostras transversais expostas a diferentes tempos de imersão em ambiente ácido.

A Figura 8, por fim, apresenta amostras longitudinais que tiveram sua fratura seccionada e polida para análise de eventuais microtrincamentos na região altamente deformada como resultado do efeito do ambiente ácido. As imagens são de elétrons retroespalhados e como pode ser evidenciado, estão presentes muito poucas inclusões alongadas na direção de laminação, independente do tempo de exposição ao meio agressivo.

* Contribuição técnica ao 69ำ Congresso Anual da ABM - Internacional e ao 14 ENEMET - Encontro Nacional de Estudantes de Engenharia Metalúrgica, de Materiais e de Minas,21 a 25 de julho de 2014, São Paulo, SP, Brasil. 

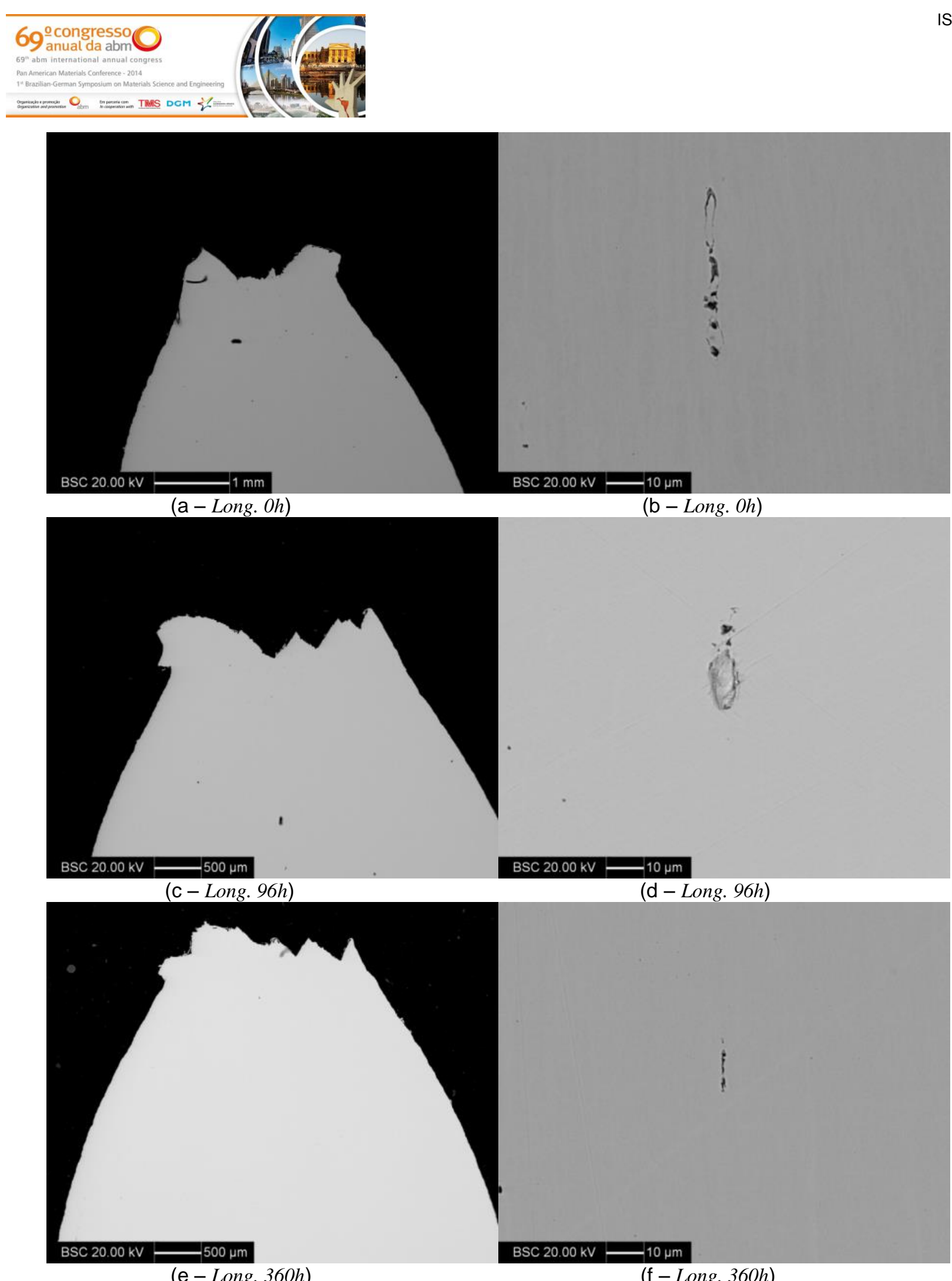

Figura 8.Seções de fraturas extraídas de amostras longitudinais expostas a diferentes tempos de imersão em ambiente ácido.

\section{CONCLUSÃO}

Não foi evidenciado no trabalho efeito deletério do ambiente ácido contendo $\mathrm{H}_{2} \mathrm{~S}$ nas propriedades mecânicas macroscópicas do aço API 5L X65 sour estudado para exposições entre 0 e 360 horas e ensaio posterior ao ataque. Somente um aumento no espalhamento experimental dos resultados das amostras atacadas foi identificado, mas

* Contribuição técnica ao 69 Congresso Anual da ABM - Internacional e ao 14ํㅡㄹ ENEMET - Encontro Nacional de Estudantes de Engenharia Metalúrgica, de Materiais e de Minas,21 a 25 de julho de 2014, São Paulo, SP, Brasil. 


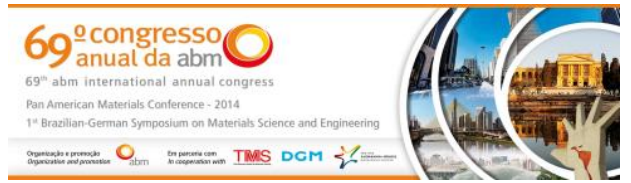

11 NACE Standard TM0284-2011. Evaluation of pipeline and pressure vessel steels for resistance to hydrogen-induced cracking. Nace International, 2011.

12 American Society for Testing and Materials -ASTM E45: Standard Test Methods for Determining the Inclusion Content of Steel. Philadelfia, USA, 2011.

13 Kyriakides S, Corona E. Pipe and Tube Manufacturing Processes, Mechanics of Offshore Pipelines, 2007.

14 Ragab,A.R.; Bayoumi, S.E.A. Engineering solid mechanics: Fundamentals and Applications. EUA: CRC Press, 1998.

15 Liou HY, Wang SC, Hsieh I. Effect of microstructures on hydrogen embrittlement and absorbed energies in low carbon steel. China steel Technical report, 1996; 10: 22-31.

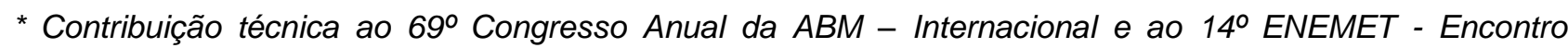
Nacional de Estudantes de Engenharia Metalúrgica, de Materiais e de Minas,21 a 25 de julho de 2014, São Paulo, SP, Brasil. 